\title{
Super Total Labeling (a,d)-Edge Antimagic on the Firecracker Graph
}

\author{
Juhari \\ Mathematics Department, Universitas Islam Negeri Maulana Malik Ibrahim Malang \\ Email: juhari@uin-malang.ac.id
}

\begin{abstract}
An An $(a, d)$-edge antimagic total labeling on $(p, q)$-graph $G$ is a one-to-one map $f$ from $V(G) \cup$ $E(G)$ onto the integers $1,2, \ldots, p+q$ with the property that the edge-weights, $w(u v)=f(u)+$ $f(v)+f(u v)$ where $u v \in E(G)$, form an arithmetic progression starting from a and having common difference $d$. Such labeling is called super if the smallest possible labels appear on the vertices. In this paper, we investigate the existence of super $(a, d)$-edge antimagic total labeling of Firecracker Graph.
\end{abstract}

Keywords: Super $(a, d)$-Edge-Antimagic Total Labeling; Firecracker Graph $\left(F_{n, k}\right)$

\section{INTRODUCTION}

The first label appears in the middle of the year 1960 it started by a Ringel and Rosa hypotheses [1]. In 1967 Rosa called this label as liberation $\beta$-valuation from a graph with e side, if there is the function that mapping one to one from the set of points $V(G)$ to set of integers $0,1,2, \ldots ., e$, so every side $\mathrm{XY}$ in graph G gets a different label $|f(x)-f(y)|$ for every edges in graph $\mathrm{G}$.

One type of graf liberation is super total labeling $(a, d)$-antimagic edge (SEATL), where a smallest side dan d different value. This liberation introduced by Simanjutak, Bertault and Miller in the year 2000 [1], [2], [3]. The entire release $(a, d)$-edge antimagic is total labeling in some kinds of graf $G$ that started by labeling all of the graphs first with consecutive original numbers, then proceed with buying all sides of the map such that the side weights form an arithmetic sequence with the first term and different $d$ [4], [5].

The type of star graph, the super total labeling $(a, d)$-edge antimagic (SEATL) not yet found one of which is a graph firecracker that hasn't been labelled previously. This prompted the writer to examine how super total labeling $(a d)$-edge antimagic (SEATL) on the firecracker graph $\left(F_{n, k}\right)$. Some of the problems formulated are as follows: (1) The upper limit $\mathrm{d}$, so the firecracker graph has super total labeling $(a, d)$-edge antimagic? And (2) How bijective function of super total labeling $(a, d)$-edge antimagic the firecracker graph?

In order not to be widespread, this research needs to be done, and this research needs to be done on total labeling $(a, d)$-edge antimagic the firecracker graph $\left(F_{n, k}\right)$ with $n \geq 2 ; k \geq 3$. In this session, $n$ and $k$ are a provision of the definition firecracker graph.

\section{Super Total Labeling $(a, d)$-Edge Antimagic.}

A graph is said to have total labeling $(a, d)$-edge antimagic if there is a one-to-one mapping of one $V(G) \cup E(G)$ to integers. $1,2,3, \ldots, p+q$ so the set of side weight $w(u v)=f(u)+$ 
$f(v)+f(u v)$ on all edge $G$ is $a, a+d, \ldots, a+(q-1) d$ for $a>0$ and $d>0$ both integers [6]. The total labeling $(a, d)$-edge antimagic called super total labeling $(a, d)$ - edge antimagic if $f(V)=\{1,2,3, \ldots, p\}$ and $f(E)=\{p+1, p+2, p+3, \ldots, p+q\}$. To search upper limit different value d super total slowly $(a, d)$-edge antimagic can certain by lemma [1], [7]:

Lemma 1 If a graph $(p, q)$ is super total labeling $(a, d)$-edge antimagic so $d \geq \frac{2 p+q-5}{q-1}$

Prove. $f(V)=\{1,2,3, \ldots, p\}$ and $f(E)=\{p+1, p+2, p+3, \ldots, p+q\}$

For example, graph $(p, q)$ is super total labeling $(a, d)$-edge antimagic by mapping $f: V(G) \cup E(G) \rightarrow\{1,2,3, \ldots, p+q\}$. The minimum value that possible from the smallest weight side $\alpha(u)+\alpha(u v)+\alpha(v)=1+(p+1)+2=p+4$ and can be written: $p+4 \leq$ $\alpha$. While on the other side, the maximum value that possible from the biggest weight side gained by the sum of 2 smallest labels and biggest label or can be written $(p-1)+$ $(p+q)+p=3 p+q-1$. Result:

$$
\begin{aligned}
a+(q-1) d & \leq 3 p+q-1 \\
d & \leq \frac{3 p+q-1-(p+4)}{q-1} \\
d & \leq \frac{2 p+q-5}{q-1}
\end{aligned}
$$

The equation above has proved and got value $d \geq \frac{2 p+q-5}{q-1}$ from many kinds or graph family.

\section{Firecracker Graph}

Firecracker graph is a graph that gets by star graph combination exactly one leaf of each graph is connected [8], [9], [10], usually symbolized $F_{n, k}$ where $n$ is the number of merged star graphs, while $k$ is the number of points of each connected star graph.

\section{METHODS}

This research uses axiomatic descriptive method, which is by decreasing the existing axioms or theorems [11], [12], then applied in super total labeling $(a, d)$-edge antimagic on the firecracker graph $F_{n, k}$. In addition, some systematic research techniques are as follows: (1) Count the number of points v and side e on the firecracker graph $F_{n, k} ;$ (2) Determine the upper limit of the different $d$ values in the firecracker graph $F_{n, k}$ in accordance with the Lemma 1; (3) determine or find EAVL label (edge-antimagic vertex labeling) or labeling points $(a, d)$ - edge antimagic of the firecracker graph $F_{n, k} ;$ (4) determine the algorithm of the functional function EAVL $f\left(x_{i . l}\right)$ on the firecracker graph $F_{n, k}$ by looking at the labeling pattern on the graph firecracker $F_{n, k}$ which has been found then grouping the numbers on the label of points that form arithmetic rows ; (5) determine the algorithm for the functional function of side weights EAVL $(w)$ on the firecracker graph $F_{n, k}$ by looking at the firecracker graph labeling pattern;(6) label the sides of the firecracker graph $F_{n, k}$ with SEATL (Super Edge Antimagic Total Labeling) or super total labeling $(a, d)$-edge antimagic for each corresponding different value $\mathrm{d} ;(7)$ Determine the bijective function on the firecracker graph $F_{n, k}$; and (8) Write a conclusion. 


\section{RESULTS AND DISCUSSION}

The first step in determining the labeling of super total $(a, d)$-edge antimagic is to determine the number of points and the number of edges on the graph under study, in this case, firecracker graph. After that, select the value of $d$ in the labeling that will be examined using lemma 1 . The labeling pattern can be determined by detecting the way (pattern recognition) after labeling a specific firecracker graph. Next, to determine patterns in general, the objective function is found by using the principle of an arithmetic sequence. The following will be presented lemma and theorems that have been found.

Lemma 2. There is a point labeling $(a, 1)$ - edge antimagic on the firecracker graph $F_{n, k}$ if $n$ odd, $n \geq 2$, and $k \geq 3$.

Prove. First defined $x_{i . l}$ is the point in the graph component of the firecracker $F_{n, k}$, where $1 \leq \mathrm{i} \leq n$ and $0 \leq \mathrm{l} \leq k-1$. Based on research results,if $\alpha: V\left(F_{n, k}\right) \rightarrow\{1,2, \ldots, n k\}$ so $\alpha$ labelation can be written as follow:

$$
\alpha\left(x_{i, l}\right)= \begin{cases}i ; & \begin{array}{l}
\text { if i odd }(1 \leq i \leq n), \\
\text { and } l=0 \\
\text { if i even }(2 \leq i \leq n-1), \\
\text { and } l=0
\end{array} \\
& \text { if i even }(12 \leq i \leq n-1), \\
& \text { and } l=1 \\
& \text { if i odd }(1 \leq i \leq n), \\
& \text { and } l=1 \\
(n+i) ; & \text { if i odd }(1 \leq i \leq n), \\
& \text { and }(2 \leq l \leq k-1) \\
n(l+1)-\frac{i-1}{2} ; & \text { if i even }(2 \leq i \leq n-1), \\
& \text { and }(2 \leq l \leq k-1) \\
& \end{cases}
$$

From the equation above $\alpha\left(x_{i, l}\right)$ is an objective function that maps $V\left(F_{n, k}\right)=$ $\left\{v_{1}, v_{2}, v_{3}, \ldots, v_{n k}\right\}$ to the set of integers $\{1,2, \ldots, n k\}$. If $w_{\alpha}$ is defindes as the weight edge of the labeling point $\alpha$, so $w_{\alpha}$ is formulated:

$w_{\alpha 1}\left(x_{i, l} x_{i, 0}\right)=(n+2 i) \quad ; 1 \leq i \leq n$, and $l=1$

$w_{\alpha 2}\left(x_{i, l} x_{i+1, l}\right)=(n+2 i+1) ; 1 \leq i \leq n-1$, and $l=1$

$w_{\alpha 3}\left(x_{i, l} x_{i, 0}\right)=(l+1) n+\frac{(i+1)}{2}$; if $i$ odd, $1 \leq i \leq n$, and $2 \leq l \leq k-1$

$w_{\alpha 4}\left(x_{i, l} x_{i, 0}\right)=(l+1) n+\frac{(n+i+1)}{2}$; if $i$ even, $1 \leq i \leq n-1$ and $2 \leq l \leq k-1$

Theorem 1. There is super total labeling $(2 n k+n+1,0)$-edge antimagic on the firecracker graph $F_{n, k}$ if $n$ odd, $n \geq 2$, and $k \geq 3$.

Prove. First define the edge label $f_{\alpha}: E\left(F_{n, k}\right)=\left\{e_{1}, e_{2}, \ldots, e_{n k-1}\right\} \rightarrow\{n k+1, n k+$ $2, \ldots, 2 n k-1\}$, so the edge label $f_{\alpha}$ for super total labeling $(a, 0)$ - edge antimagic on the graph $F_{n, k}$ can be formulated as follows:

$f_{\alpha 1}\left(x_{i, l} x_{i, 0}\right)=2(n k-i)+1 \quad ; 1 \leq i \leq n$ and $l=1$

$f_{\alpha 2}\left(x_{i, l} x_{i+1, l}\right)=2(n k-i) \quad ; 1 \leq i \leq n-1$ and $l=1$

$f_{\alpha 3}\left(x_{i, l} x_{i, 0}\right)=(2 k-l) n+\frac{(1-i)}{2} \quad ;$ if $i$ odd, $1 \leq i \leq n$, and $2 \leq l \leq k-1$

$f_{\alpha 4}\left(x_{i, l} x_{i, 0}\right)=(2 k-l) n+\frac{(-2 n-i+6)}{2}$; if $i$ even, $2 \leq i \leq n-1$, and $2 \leq l \leq k-1$

Next, if there is $W_{\alpha}$ even that defined as weight of super total labeling 
$\alpha\left(x_{i, l}\right), \alpha\left(x_{i, l} x_{i, 0}\right)$, and $\alpha\left(x_{i, l} x_{i+1, l}\right)$, so $W_{\alpha}$ can be obtained by adding up the edge weight formula EAVL $w_{\alpha}$ and the formula of edge label $f_{\alpha}$ with the terms of boundaries $i$ and $l$ which correspond and can be stated as follows:

$\begin{array}{ll}W_{\alpha 1}=w_{\alpha 1}\left(x_{i, l} x_{i, 0}\right)+f_{\alpha 1}\left(x_{i, l} x_{i, 0}\right) & ; 1 \leq i \leq n, \text { and } l=1 \\ W_{\alpha 2}=w_{\alpha 2}\left(x_{i, l} x_{i, 0}\right)+f_{\alpha 2}\left(x_{i, l} x_{i, 0}\right) & ; 1 \leq i \leq n-1, \text { and } l=1 \\ W_{\alpha 3}=w_{\alpha 3}\left(x_{i, l} x_{i+1, l}\right)+f_{\alpha 3}\left(x_{i, l} x_{i+1, l}\right) & ; \text { if } i \text { odd, } 1 \leq i \leq n, \text { and } 2 \leq l \leq k-1 \\ W_{\alpha 4}=w_{\alpha 3}\left(x_{i, l} x_{i+1, l}\right)+f_{\alpha 3}\left(x_{i, l} x_{i+1, l}\right) & ; \text { if } i \text { even, } 2 \leq i \leq n-1, \text { and } 2 \leq l \leq k-1\end{array}$

By substituting the equation above is obtained:

$$
\begin{aligned}
W_{\alpha 1} & =(n+2 i)+2(n k-i)+1 \\
& =2 n k+n+1 \\
W_{\alpha 2} & =2(n k-i)+(n+2 i+1) \\
& =2 n k+n+1 \\
W_{\alpha 3} & =(2 k-l) n+\frac{(1-i)}{2}+(l+1) n+\frac{(i+1)}{2} \\
& =2 n k+n+1 \\
W_{\alpha 4} & =(2 k-l) n+\frac{(-2 n-i+6)}{2}+(l+1) n+\frac{(n+i+1)}{2} \\
& =2 n k+n+1
\end{aligned}
$$

Based on the equation above, the set of total labeling edge weights can be written as $W_{\alpha}=\left\{W_{\alpha 1}, W_{\alpha 2}, W_{\alpha 3}, W_{\alpha 4}\right\}$. It can also be seen that $W_{\alpha 1}=W_{\alpha 2}=\cdots=W_{\alpha 4}=2 n k+$ $n+1$ or can be written as follows: $\bigcup_{t=1}^{4} W_{\alpha t}=\{2 n k+n+1,2 n k+n+1, \ldots, 2 n k+n+$ $1\}$. From this it can be concluded that the firecracker graph $F_{n, k}$ have Super $(2 n k+n+$ $1,0)$ - edge antimagic if $n$ odd, $n \geq 2$, and $k \geq 3$.

Theorem 2. There are super total labeling $((k+1) n+3,2)$-edge antimagic on the graph firecracker $F_{n, k}$ if $n$ odd $(n \geq 2)$, and $k \geq 3$.

Prove. First define the edge label $f_{\alpha}: E\left(F_{n, k}\right)=\left\{e_{1}, e_{2}, \ldots, e_{n k-1}\right\} \rightarrow\{n k+1, n k+$ $2, \ldots, 2 n k-1\}$, so edge label $f_{\alpha}$ for super total labeling $(a, 2)$ - edge antimagic on the graph $F_{n, k}$ can be formulated as follows:

$$
\begin{array}{ll}
f_{\alpha 1}\left(x_{i, l} x_{i, 0}\right)=(n k+2 i-1) & ; 1 \leq i \leq n \text { and } l=1 \\
f_{\alpha 2}\left(x_{i, l} x_{i+1, l}\right)=(n k+2 i) & ; 1 \leq i \leq n-1 \text { and } l=1 \\
f_{\alpha 3}\left(x_{i, l} x_{i, 0}\right)=(k+l) n+\frac{(i-1)}{2} & ; \text { if } i \text { odd, } 1 \leq i \leq n, \text { and } 2 \leq l \leq k-1 \\
f_{\alpha 4}\left(x_{i, l} x_{i, 0}\right)=(k+l) n+\frac{(n+i-1)}{2} & ; \text { if } i \text { even, } 2 \leq i \leq n-1 \text {, and } 2 \leq l \leq k-1
\end{array}
$$

Next, $W \alpha$ defined as weight of super total labeling edge $\alpha\left(x_{i, l}\right), \alpha\left(x_{i, l} x_{i, 0}\right)$, and $\alpha\left(x_{i, l} x_{i+1, l}\right)$

$W_{\alpha 1}=w_{\alpha 1}\left(x_{i, l} x_{i, 0}\right)+f_{\alpha 1}\left(x_{i, l} x_{i, 0}\right) \quad ; 1 \leq i \leq n$, and $l=1$

$W_{\alpha 2}=w_{\alpha 2}\left(x_{i, l} x_{i, 0}\right)+f_{\alpha 2}\left(x_{i, l} x_{i, 0}\right) \quad ; 1 \leq i \leq n-1$, and $l=1$

$W_{\alpha 3}=w_{\alpha 3}\left(x_{i, l} x_{i+1, l}\right)+f_{\alpha 3}\left(x_{i, l} x_{i+1, l}\right) ;$ if $i$ odd, $1 \leq i \leq n$, and $2 \leq l \leq k-1$

$W_{\alpha 4}=w_{\alpha 3}\left(x_{i, l} x_{i+1, l}\right)+f_{\alpha 3}\left(x_{i, l} x_{i+1, l}\right)$; if $i$ even, $2 \leq i \leq n-1$, and $2 \leq l \leq k-1$

By substituting the equation above is obtained:

$$
\begin{aligned}
W_{\alpha 1} & =(n+2 i)+(n k+2 i-1) \\
& =(k+1) n+4 i-1 \\
W_{\alpha 2} & =(n+2 i+1)+(n k+2 i) \\
& =(k+1) n+4 i+1 \\
W_{\alpha 3} & =(l+1) n+\frac{(i+1)}{2}+(k+l) n+\frac{(i-1)}{2}
\end{aligned}
$$




$$
\begin{aligned}
& =(k+2 l+1) n+i \\
W_{\alpha 4} & =(l+1) n+\frac{(n+i+1)}{2}+(k+l) n+\frac{(n+i-1)}{2} \\
& =(k+2 l+2) n+i
\end{aligned}
$$

Based on the equation above, the set of total labeling edge weights can be written as $W_{\alpha}=\left\{W_{\alpha 1}, W_{\alpha 2}, W_{\alpha 3}, W_{\alpha 4}\right\}$. It can also be seen that $W_{\alpha 1}=W_{\alpha 2}=\cdots=W_{\alpha 4}=2 n k+$ $n+1$ or can be written as follows: $\bigcup_{t=1}^{4} W_{\alpha t}=\{(k+1) n+3,(k+1) n+5, \ldots,(3 k+$ $1) n-1\}$. From this it can be concluded that the firecracker graph $F_{n, k}$ have Super $((k+1) n+3,2)$ - edge antimagic if $n$ odd, $n \geq 2$, and $k \geq 3$.

Theorem 3. There is super total labeling $(2 n k+1,1)$-edge antimagic on the graph firecracker $F_{n, k}$ if $n$ even $(n \geq 2)$, and $k \geq 3$.

Prove. To determine the total super labeling $(a, 1)$-edge antimagic, defined first $f_{a}: E\left(F_{n, k}\right)=\left\{e_{1}, e_{2}, \ldots, e_{n k-1}\right\} \rightarrow\{n k+1, n k+2, \ldots, 2 n k-1\}$ which is the labeling edge label and can be formulated:

$$
\begin{aligned}
& f_{\alpha 1}\left(x_{i, l} x_{i, 0}\right)=(2 n k-4 i+2) \quad ; 1 \leq i \leq n \text { and } l=1 \\
& f_{\alpha 2}\left(x_{i, l} x_{i+1, l}\right)=(2 n k-4 i) \quad ; 1 \leq i \leq n-1 \text { and } l=1 \\
& f_{\alpha 3}\left(x_{i, l} x_{i, 0}\right)=(2 k-l) n+3 n-i \quad ; \text { if } i \text { odd, } 1 \leq i \leq n \text {, and } 2 \leq l \leq k-1 \\
& f_{\alpha 4}\left(x_{i, l} x_{i, 0}\right)=(2 k-l) n+2 n-1 \text {; if } i \text { even, } 2 \leq i \leq n-1 \text {, and } 2 \leq l \leq k-1 \\
& \text { If } W_{\alpha} \text { defined as the total labeling edge weight } \alpha\left(x_{i, l}\right), \alpha\left(x_{i, l} x_{i, 0}\right) \text {, and } \alpha\left(x_{i, l} x_{i+1, l}\right) \text {. } \\
& W_{\alpha 1}=w_{\alpha 1}\left(x_{i, l} x_{i, 0}\right)+f_{\alpha 1}\left(x_{i, l} x_{i, 0}\right) \quad ; 1 \leq i \leq n \text {, and } l=1 \\
& W_{\alpha 2}=w_{\alpha 2}\left(x_{i, l} x_{i, 0}\right)+f_{\alpha 2}\left(x_{i, l} x_{i, 0}\right) \quad ; 1 \leq i \leq n-1 \text {, and } l=1 \\
& W_{\alpha 3}=w_{\alpha 3}\left(x_{i, l} x_{i+1, l}\right)+f_{\alpha 3}\left(x_{i, l} x_{i+1, l}\right) ; \text { if } i \text { odd, } 1 \leq i \leq n \text {, and } 2 \leq l \leq k-1 \\
& W_{\alpha 4}=w_{\alpha 3}\left(x_{i, l} x_{i+1, l}\right)+f_{\alpha 3}\left(x_{i, l} x_{i+1, l}\right) \text {; if } i \text { even, } 2 \leq i \leq n-1 \text {,and } 2 \leq l \leq k-1 \\
& \text { By substituting the equation above is obtained: } \\
& \begin{aligned}
W_{\alpha 1} & =(n+2 i)+(2 n k-4 i+2) \\
& =(2 n k+n-2 i+2) \\
W_{\alpha 2} & =(2 n k-4 i)+(n+2 i+1) \\
& =(2 n k+n-2 i+1) \\
W_{\alpha 3} & =(2 k-l) n+3 n-i+(l+1) n+\frac{(i+1)}{2} \\
& =\left(2 n k+4 n+\frac{(i+1)}{2}\right) \\
W_{\alpha 4} & =(2 k-l) n+2 n-i+(l+1) n+\frac{(n+i+1)}{2} \\
& =\left(2 n k+\frac{7 n+1-i}{2}\right)
\end{aligned}
\end{aligned}
$$

Based on the equation above, the set of total labeling edge weights can be witten with $W_{\alpha}=\left\{W_{\alpha 1}, W_{\alpha 2}, W_{\alpha 3}, W_{\alpha 4}\right\}$. It can also be seen that the smalest edge lies in $W_{\alpha 2}$ and the biggest edge weights lies in $W_{\alpha 1}$, can be stated that $W_{\alpha}$ forming arithmetic lines with initial term $2 n k+1$ and different 1 (one), or can be written $\bigcup_{t=1}^{4} W_{\alpha t}=\{2 n k+1,2 n k+$ $2,2 n k+3, \ldots,((3 n k-1)+i)\}$. So, can be conclude that the firecracker graph $F_{n, k}$ have Super $(2 n k+1,1)$-EAT; n even $(n \geq 2)$, and $k \geq 3$. 
Theorem 4. There is super total labeling $\{n k+n+4,3\}$-edge antimagic on the combination of firecracker graph $m F_{n k}$ if $m \geq 2$, n even $(n \geq 2)$, and $n \geq 3$.

Prove. To determine the total super labeling $(a, 1)$-edge antimagic, defined first $f_{a}: E\left(F_{n, k}\right)=\left\{e_{1}, e_{2}, \ldots, e_{n k-1}\right\} \rightarrow\{n k+1, n k+2, \ldots, 2 n k-1\}$ which is the edge label and can be formulated:

$$
\begin{aligned}
& f_{\alpha 1}\left(x_{i, l} x_{i, 0}\right)=(n k+4 i-2) \quad ; 1 \leq i \leq n \text { and } l=1 \\
& f_{\alpha 2}\left(x_{i, l} x_{i+1, l}\right)=(n k+4 i) \quad ; 1 \leq i \leq n-1 \text { and } l=1 \\
& f_{\alpha 3}\left(x_{i, l} x_{i, 0}\right)=(2 k-l) n+i-1 \quad \text {; if } i \text { odd, } 1 \leq i \leq n \text {, and } 2 \leq l \leq k-1 \\
& f_{\alpha 4}\left(x_{i, l} x_{i, 0}\right)=(2 k-l) n+n+i-1 \text {; if } i \text { even, } 2 \leq i \leq n-1 \text {, and } 2 \leq l \leq k-1 \\
& \text { If } W_{\alpha} \text { defined as the total edge labeling weights } \alpha\left(x_{i, l}\right), \alpha\left(x_{i, l} x_{i, 0}\right) \text {, and } \alpha\left(x_{i, l} x_{i+1, l}\right) \text {. } \\
& W_{\alpha 1}=w_{\alpha 1}\left(x_{i, l} x_{i, 0}\right)+f_{\alpha 1}\left(x_{i, l} x_{i, 0}\right) \quad ; 1 \leq i \leq n, \text { and } l=1 \\
& W_{\alpha 2}=w_{\alpha 2}\left(x_{i, l} x_{i, 0}\right)+f_{\alpha 2}\left(x_{i, l} x_{i, 0}\right) \quad ; 1 \leq i \leq n-1 \text {, and } l=1 \\
& W_{\alpha 3}=w_{\alpha 3}\left(x_{i, l} x_{i+1, l}\right)+f_{\alpha 3}\left(x_{i, l} x_{i+1, l}\right) ; \text { if } i \text { odd, } 1 \leq i \leq n \text {, and } 2 \leq l \leq k-1 \\
& W_{\alpha 4}=w_{\alpha 3}\left(x_{i, l} x_{i+1, l}\right)+f_{\alpha 3}\left(x_{i, l} x_{i+1, l}\right) \text {; if } i \text { even, } 2 \leq i \leq n-1 \text {, and } 2 \leq l \leq k-1 \\
& \text { By substituting the equation above is obtained: } \\
& W_{\alpha 1}=(n+2 i)+(n k+4 i+2) \\
& =(n k+n+6 i+2) \\
& W_{\alpha 2}=(n k+2 i+1)+(n k+4 i) \\
& =(2 n k+n+6 i+1) \\
& W_{\alpha 3}=(l+1) n+\frac{(i+1)}{2}+(2 k-l) n+i-1 \\
& =\left(2 n k+n+\frac{(1-i)}{2}\right) \\
& W_{\alpha 4}=(2 k-l) n+n-i+1+(l+1) n+\frac{(n+i+1)}{2} \\
& =\left(2 n k+\frac{5 n+2 i-1}{2}\right)
\end{aligned}
$$

Based on the equation above, the set of total labeling edge weights can be written with $W_{\alpha}=\left\{W_{\alpha 1}, W_{\alpha 2}, W_{\alpha 3}, W_{\alpha 4}\right\}$. It can also be seen that the smalest edge lies in $W_{\alpha 1}$ and the biggest edge weights lies in $W_{\alpha 2}$, can be stated that $W_{\alpha}$ forming arithmetic lines with initial term $n k+n+4$ and different 3 (one), or can be written $\bigcup_{t=1}^{4} W_{\alpha t}=\{n k+n+4, n k+$ $n+7, n k+n+10, \ldots,((3 n k+1) n+i)\}$. So, can be conclude that the firecracker graph $F_{n, k}$ have Super $(2 n k+1,1)$-EAT; $n$ even $(n \geq 2)$, and $k \geq 3$.

\section{CONCLUSIONS}

Based on the result, can be concluded that firecracker graph $F_{n, k}$ have super total labeling $(a, d)$-edge antimagic, with $d \in\{0,1,2,3\}$ and bijective function in some of Lemma and Theorem show about super complete labeling $(a, d)$-edge antimagic on the firecracker graph. The bijective function for each liberation with $d$ different value have shown in equation (1),(2),(3) until (10) above.

Open problem: super total labeling $(a, d)$-edge antimagic on the firecracker graph $F_{n, k}$ for $\mathrm{d}=0$ and $\mathrm{d}=2$ where $n$ even and $k \geq 3$; super total labeling $\mathrm{d}=1$ and $\mathrm{d}=3$ where $\mathrm{n}$ odd and $k \geq 3$. 


\section{REFERENCES}

[1] Dafik, "Structural Properties and Labeling of Graphs Statement of Authorship," no. November, pp. 1-139, 2007.

[2] M. Baca, L. Brankovic, M. Lascsakova, O. Phanalasy, and A. SemanicovaFenovclkova, "On d-Antimagic Labelings of Plane Graphs," Electron. J. Graph Theory Appl., vol. 1, no. 1, pp. 28-39, 2013.

[3] M. A. Muttaqien and A. Suyitno, "Pelabelan Total Sisi Ajaib Pada Graf Double Star dan Graf Sun,” Unnes J. Math., vol. 2, no. 2, 2013.

[4] R. H. Utomo, H. Tjahjana, B. Irawanto, and L. Ratnasari, "Pelabelan Total Super Trimagic Sisi Pada Beberapa Graf,” J. Fundam. Math. Appl., vol. 1, no. 1, p. 52, Jun. 2018.

[5] T. Utomo and N. Riskiana Dewi, "Dimensi Metrik Graf Amal (nKm)," Limits J. Math. Its Appl., vol. 15, no. 1, p. 71, Mar. 2018.

[6] V. Swaminathan and P. Jeyanthi, "Super edge-magic strength of firecrackers, banana trees and unicyclic graphs," Discrete Math., vol. 306, no. 14, pp. 16241636, 2006.

[7] S. Chattopadhyay and P. Panigrahi, "Some structural properties of power graphs and k -power graphs of finite semigroups," J. Discret. Math. Sci. Cryptogr., vol. 20, no. 5, pp. 1101-1119, Jul. 2017.

[8] Y.-N. Chen, W.-C.; Lu, H.-I; and Yeh, "Operations of Interlaced Trees and Graceful Trees," Southeast Asian Bull. Math, vol. 21, pp. 337-348, 1997.

[9] J. A. Gallian, "A dynamic survey of graph labeling," Electron. J. Comb., vol. 1, no. DynamicSurveys, pp. 1-256, 2018.

[10] K. A. Sugeng and D. R. Silaban, "On b-edge consecutive edge labeling of some regular tree," Indones. J. Comb., vol. 4, no. 1, p. 76, 2020.

[11] I. Halikin, "Pelabelan Lokal Titik Graf Hasil Diagram Lattice Subgrup Zn," AlKhwarizmi J. Pendidik. Mat. dan Ilmu Pengetah. Alam, vol. 6, no. 1, pp. 47-56, Mar. 2018.

[12] A. S. Meliana Deta Anggraeni, Mulyono, "Pelabelan L(3,2,1) dan Pembentukan Graf Middle Pada Beberapa Graf Khusus,” vol. 2, no. 1, pp. 76-83, 2013. 